\title{
Energy Radiated by Accelerated Electrons
}

\author{
Ardeshir Irani \\ The Dark Energy Research Institute, Downey, CA, USA \\ Email: artirani@aol.com
}

How to cite this paper: Irani, A. (2022) Energy Radiated by Accelerated Electrons. Journal of High Energy Physics, Gravitation and Cosmology, 8, 143-146. https://doi.org/10.4236/jhepgc.2022.81010

Received: November 5, 2021

Accepted: January 2, 2022

Published: January 5, 2022

Copyright $\odot 2022$ by author(s) and Scientific Research Publishing Inc. This work is licensed under the Creative Commons Attribution International License (CC BY 4.0).

http://creativecommons.org/licenses/by/4.0/

\begin{abstract}
The Law of Lepton Conservation Number tells us that whenever electrons radiate energy in the form of photons, the photon radiation must be accompanied by electron neutrinos. Synchrotrons such as the National Synchrotron Light Source (NSLS 11) at Brookhaven National Laboratory or the Stanford Synchrotron Radiation Light Source (SSRL) would be excellent sources for producing abundant neutrinos for neutrino detection studies. Bremsstrahlung (electron breaking mechanism) is the process that explains energy jets as observed being given out from Active Galactic Nuclei and Pulsars.
\end{abstract}

\section{Keywords}

The Law of Lepton Conservation Number, Neutrinos, Synchrotrons

\section{Lepton Number Conservation Law}

The Lepton number on the left side of the equation must equal the Lepton number on the right side of the equation. Electrons and electron neutrinos are given the number +1 . Positrons and positron antineutrinos are given -1 . All other particles including photons are given 0 .

Electron (1) $\rightarrow$ Electron neutrino (1) + photons (0),

Positron $(-1) \rightarrow$ Positron antineutrino $(-1)+$ photons $(0)$.

For each electron only one neutrino can be produced and for each positron only 1 antineutrino can be produced to balance the numbers in the above equations. Hence the need for photons to conserve Energy because the mass of the neutrino/antineutrino is infinitesimally small compared to the mass of the electron/positron.

This law implies that when accelerated electrons/positrons radiate energy in the form of photons some of the energy is carried away by neutrinos/antineutrinos that are equal in number to the number of radiating electrons/positrons.

There is no significant amount of radiation for non-relativistic motion be- 
cause the energy increase goes into changing the velocity of the electron. There is significant radiation for ultra-relativistic motion because there is a limit on the speed that can be achieved which is the speed of light. Hence most of the energy put in must be radiated away since the electron can no longer change its speed. The photons and electron neutrinos created by the energy given to the electrons would shoot out in the direction of the acceleration or the direction of forward electron motion. Experimentally accelerators such as the National Synchrotron Light source at Brookhaven National Laboratory (BNL) or the Stanford Synchrotron Radiation Light Source (SSRL) would be excellent measuring tools for neutrinos because they have the capacity of creating so many of them.

A similar process would apply to accelerated positrons which would be an excellent measuring tool for antineutrinos.

By placing neutrino/antineutrino detectors closer to their source the probability of detecting them becomes much greater.

The same conservation law would also apply to muon-leptons and tau-leptons.

National Synchrotron Light Source NSLS 11 (BNL) in Long Island, New York:

For NSLS 11 the Accelerator Parameters are (3):

Energy $E=3 \mathrm{GeV}$, Current $I=400 \mathrm{~mA}$, Circumference $=792 \mathrm{~m}$, Radius $\rho=$ $792 / 2 \pi=126.1 \mathrm{~m}$, Energy Lost per revolution $=1767.3 \mathrm{keV}$, Number of bunches $=1056$, Average Bunch Charge $=1.25 \mathrm{nC}$, Total Charge $Q=1056 \times 1.25 \mathrm{nC}=$ $1.32 \mu \mathrm{C}$.

For high energy electrons $(\beta=1)$ the radiative energy loss and the power radiated per revolution for an incoherent circulating electron beam of current $I$ are given by [1]:

$$
\begin{gathered}
\left.\delta E(\mathrm{MeV})=8.85 \times 10^{-12}\left[E(\mathrm{GeV})^{4}\right] / \rho \text { (meters }\right) \\
\left.P(\text { Watts })=10^{6} \delta E(\mathrm{MeV}) I(\text { Amps }) /\{2 \pi \rho \text { (meters })\right\}
\end{gathered}
$$

Plugging in the numbers for NSLS 11 into the above two equations we get:

$$
\begin{gathered}
\delta E(\mathrm{MeV})=0.0568 \mathrm{MeV} \text { or } 56.8 \mathrm{keV} \\
P(\text { Watts })=28.3 \text { Watts }
\end{gathered}
$$

These numbers would correspond to only the partial loss per revolution, the total measured value being $1767.3 \mathrm{keV}$ since they do not include radiation emitted by Wiggler magnets placed within the machine where coherent radiation is possible [2], energy taken away by electron neutrinos, and the efficiency of the synchrotron since no machine is $100 \%$ efficient. For incoherent radiation the energy radiated is proportional to $n_{B}$ where $n_{B}$ represents the total number of electrons in a bunch of the beam while for coherent radiation it is proportional to $n_{B}^{2}[2]$.

The number of electrons $=n e=Q$ where " $e$ " is the charge of one electron. Hence $n=8.25 \times 10^{12}$. Since each electron can only create one electron neutrino to satisfy the Lepton Number Conservation Law, $n_{v}=8.25 \times 10^{12}$ neutrinos would be emitted per revolution every time the electrons receive an energy boost 
to make up for the energy loss per turn.

Stanford Synchrotron Radiation Light Source (SSRL) in Menlo Park, California.

For SSRL SPEAR 3 the Accelerator Parameters are [3]:

$E=3 \mathrm{GeV}, I=500 \mathrm{~mA}$, Circumference $=234 \mathrm{~m}$, Radius $\rho=37.3 \mathrm{~m}, Q=0.39$ $\mu \mathrm{C}$.

Plugging in these numbers we get:

The total number of electrons $n$ and therefore electron neutrinos that are produced per revolution $n_{v}=2.44 \times 10^{12}$.

$$
\begin{gathered}
\delta E(\mathrm{MeV})=0.19 \mathrm{MeV} \text { or } 190 \mathrm{keV} . \\
P(\text { Watts })=405.6 \text { Watts } .
\end{gathered}
$$

The Energy and Power loss per revolution is greater in this case than in the case of NSLS 11 because of the smaller radius of SSRL SPEAR 3.

\section{Bremsstrahlung}

The radiation emitted during atomic collisions is customarily called bremsstrahlung (breaking radiation) because it was first observed when high energy electrons were stopped in a thick metallic target. For non-relativistic electrons the loss of energy by radiation is negligible compared with the collisional energy loss, but for relativistic electrons radiation is the dominant mode of energy loss.

Active Galactic Nuclei are the neutrinos and photons radiated out of the Supermassive Black Hole at the center of a Galaxy in the form of jets due to Bremsstrahlung as electrons encounter matter sucked in from the accretion disk of the Black Hole and are slowed down by their interaction with these particles. The acceleration is in the opposite direction to the electron velocity and hence the radiation (neutrinos and photons) created by the energy taken out of the electron motion is in the direction opposite to the matter being sucked into the Black Hole.

We have proven [4] that a neutron star would have to be rotating at a fast enough critical speed for its magnetic field to provide its mass with enough energy to create a Black Hole. Jets from Pulsars (neutron stars that have not yet attained the requisite speed) are proof that the Bremsstrahlung process starts up even before the rotating neutron stars are able to create a Black Hole because the space-time curvature created by the Pulsar is large enough to suck in matter to slow down the electrons as in the case of the Black Hole.

\section{Conflicts of Interest}

The author declares no conflicts of interest regarding the publication of this paper.

\section{References}

[1] Jackson, J.D. (1962) Classical Electrodynamics. John Wiley \& Sons, Inc., Hoboken. 
Equations 14.33, 14.34, p. 471, 472.

[2] Irani A. (1979) BNL-26690, Synchrotron Radiation from a Helical Wiggler. Brookhaven National Laboratory, Upton, N.Y. 11973 I.

[3] Accelerator Parameters for NSLS 11 and SSRL SPEAR 3 Are Obtained from the Internet by Googling National Synchrotron Light Source and Stanford Synchrotron Radiation Light Source.

[4] Irani A. (2022) Stellar Rotating Black Holes. Journal of High Energy Physics, Gravitation and Cosmology, 8, 97-99. https://doi.org/10.4236/jhepgc.2022.81007 